\title{
Raumforschung und Raumordnung - Spektrum raumwissenschaftlicher Forschung
}

\author{
Andreas Klee
}

Online publiziert: 20. Januar 2012

(C) Springer-Verlag 2012

Demographischer Wandel, seit geraumer Zeit ein wichtiges Thema in Forschung und Praxis, war schon häufig ein Sujet in den Heften der Zeitschrift ,Raumforschung und Raumordnung". Analysiert man die Beiträge der vergangenen zehn Jahre, erkennt man jedoch schnell, dass sich die Herangehensweise an das Thema verändert hat. Wurden zunächst vor allem Beiträge veröffentlicht, die sich in analytischer oder konzeptioneller Weise mit der Alterung und dem Rückgang der Bevölkerung in Deutschland und Europa sowie mit ihrer sozialen Durchmischung befassten, so hat sich in den letzten Jahren der Fokus etwas verschoben. Fragen, wie Städte und Regionen mit dem Wandel umgehen oder umgehen sollten, gewannen ebenso an Bedeutung wie regionale oder sektorale Fallstudien. Daraus lässt sich ableiten, dass das Problembewusstsein nicht nur in der Wissenschaftsgemeinschaft, sondern auch bei Planungspraktikern und Politikern gestiegen ist, und dass Strategien nachgefragt werden, wie der demographische Wandel zu bewältigen oder zu begleiten ist.

Eng mit dem demographischen Wandel verbunden ist ein Faktor, der in der raumwissenschaftlichen Diskussion häufig zu kurz kommt: die Mortalität. Aus raumwissenschaftlicher Sicht verwundert dies, denn auch die Sterblichkeitsverhältnisse haben sich in den letzten Dekaden gewandelt und weisen nicht zu vernachlässigende regionale Besonderheiten auf. Im vorliegenden Heft führt Holger Behrendt eine Regionalanalyse der Mortalität in Deutschland für die Jahre 1998 bis 2004 durch. Der dabei ermittelte „Aufholprozess“ der neuen Bundesländer gegenüber den westdeutschen

Dr. A. Klee $(\bowtie)$

Akademie für Raumforschung und Landesplanung Leibniz-Forum für Raumwissenschaften,

Hohenzollernstraße 11, 30161 Hannover, Deutschland

E-Mail: klee@arl-net.de
Sterblichkeitsverhältnissen mag nicht verwundern. Dass jedoch eine in Ost- und Westdeutschland unterschiedliche kleinräumige (siedlungsstrukturelle) Differenzierung der Mortalität festgestellt werden kann, legt nahe, das bisher vorherrschende großräumige Denken sowie den dominanten Einfluss sozioökonomischer Faktoren zu relativieren. Darüber hinaus wird klar, dass die festgestellten regionalen Unterschiede in den Sterblichkeitsverhältnissen den demographischen Wandel hinsichtlich seiner regional und kommunal bedeutsamen Ausprägungen weiter differenzieren.

Für einen Zeitraum von vier Jahrzehnten (1961-2001) untersuchen Matthias Kranabether, Marco Helbich und Hermann Knoflacher Zusammenhänge zwischen dem Ausbaugrad von Autobahnen und Schnellstraßen einerseits und der Anzahl nichtlandwirtschaftlicher Arbeitsstätten andererseits. Für ihre Studie zu raumstrukturellen Aspekten des Fernstraßenbaus in der Ostregion in Österreich arbeiteten sie am Beispiel der österreichischen Bundesländer Wien, Niederösterreich und Burgenland. Die Autoren konnten feststellen, dass die „Gewinner“" an Arbeitsstätten vor allem jene Gemeinden sind, die im unmittelbaren Einzugsbereich des Großraums Wien liegen. Demgegenüber gibt es - insbesondere in jüngerer Zeit - eine wachsende Zahl an Gemeinden, die, zum Teil trotz guter Straßenverkehrsanbindung, keine überdurchschnittlichen Zuwachsraten aufweisen. Welche Schlüsse ziehen die Autoren unter anderem daraus? Offenbar ist in infrastrukturell bereits gut entwickelten Staaten und Regionen kein großräumiger Nutzen hinsichtlich der Entstehung von Arbeitsstätten mehr zu erwarten. Anders ausgedrückt: Straßenbau (allein) schafft keine neuen Arbeitsplätze. Die Nähe zu prosperierenden Zentren ist wesentlich bedeutsamer. Dies ist keine neue Erkenntnis - allerdings eine neue, empirisch fundierte Bestätigung der Ergebnisse zahlreicher Studien. 
Einen anderen, ebenso interessanten Zusammenhang beleuchtet Franz-Josef Bade in seinem Beitrag, in dem er nach dem Erfolg der durch die Gemeinschaftsaufgabe „Verbesserung der regionalen Wirtschaftsstruktur" geförderten Betriebe fragt, damit also nach der Wirkung von Subventionen auf das Innovationsverhalten von Betrieben. Sein Fazit ist eindeutig: Die geförderten Betriebe leisten einen erheblichen Beitrag zur Beschäftigung in strukturschwachen Regionen. Diesem Ergebnis liegt eine breit angelegte quantitative Untersuchung in den Jahren 1998 bis $2008 \mathrm{zu}$ Grunde. $\mathrm{Ob}$ das Ergebnis allerdings auch als Beleg für die Effizienz der deutschen Regionalförderung gewertet werden soll, ist umstritten, was auch der Autor betont. Gleichwohl wird hervorgehoben, dass die Investitionsförderung im Rahmen der Gemeinschaftsaufgabe dazu beitragen kann, Beschäftigung zu schaffen und zu sichern sowie regionale Wertschöpfung zu erhöhen.

Beschäftigung spielt auch eine Rolle im Beitrag von Alexander Werner und Reinhold Kosfeld. Sie legen ein Verfahren sowie die Ergebnisse einer Neuabgrenzung deutscher Arbeitsmarktregionen nach den jüngsten Kreisgebietsreformen vor. Ihre Untersuchung basiert auf einer Faktorenanalyse und resultiert in 141 regionalen Arbeitsmarktregionen in Deutschland. Übrigens werden in „Raumforschung und Raumordnung“ immer wieder Vorschläge zur Abgrenzung von Arbeitsmarktregionen vorgestellt und diskutiert - auch ein Beleg für die Relevanz regionalökonomischer Fragestellungen in der breiten raumwissenschaftlichen Forschung.

Schließlich stellen Ulrike Weiland, Andreas Klee, Jörg Knieling und Dietmar Scholich in ihrem Beitrag das Mentoring-Programm der Akademie für Raumforschung und Landesplanung (ARL) und des Förderkreises für Raum- und Umweltforschung (FRU) vor. Das Ziel des Programmes ist es, junge Frauen aus Wissenschaft und Praxis der Raumund Umweltplanung bei ihrer beruflichen Entwicklung zu unterstützen. Dies soll dazu beitragen, die Nachwuchsförderung strategischer zu gestalten und damit den Anteil von Frauen in leitenden Funktionen zu erhöhen. Mittelpunkt des Artikels ist die Auswertung einer Befragung von ehemaligen Teilnehmerinnen des Programms sowie hieraus die Ableitung von Schlussfolgerungen.

Das Jahr 2012 bringt für die Herausgeber der Zeitschrift „Raumforschung und Raumordnung" eine Neuerung mit sich. Nach zwei Jahren Tätigkeit als Editor-in-Chief ist Gregor Prinzensing Ende 2011 turnusmäßig ausgeschieden. Seine Nachfolgerin - an der Seite von Andreas Klee - ist Hendrikje Wehnert vom Leibniz-Institut für ökologische Raumentwicklung in Dresden. Die Herausgeber danken Gregor Prinzensing für seine Tätigkeit, die er mit viel Umsicht, Einfühlungsvermögen, Verlässlichkeit und Kompetenz ausgeführt hat, und wünschen Hendrikje Wehnert viel Freude und Erfolg. 